\title{
Famílias e síndrome de Down: Estresse, coping e recursos familiares
}

\author{
Nara Liana Pereira-Silva*, Jaqueline Condé Melo Andrade, \& Bruna Rocha Almeida \\ Universidade Federal de Juiz de Fora. Juiz de Fora, MG, Brasil.
}

\begin{abstract}
RESUMO - Este estudo objetiva identificar níveis de estresse e coping - ou estratégias de enfrentamento - de genitores de crianças com síndrome de Down (SD), bem como a adequação dos recursos familiares. Quatorze famílias com um(a) filho(a) com SD foram visitadas e os genitores responderam ao questionário de caracterização do sistema familiar, inventário de estresse, inventário de coping e escala de recursos familiares. Os resultados mostram que mães $(n=7)$ e pais $(n=6)$ apresentam sintomas de estresse, nas fases de resistência e quase exaustão. As estratégias de enfrentamento mais utilizadas pelos genitores são resolução de problemas e suporte social. Os dados da escala de recursos indicam certa adequação destes às necessidades das famílias. Ressalta-se a importância de programas de intervenção dirigidos a essas famílias. PALAVRAS-CHAVE: estratégias de enfrentamento, síndrome de Down, família, apoio social, recursos familiares
\end{abstract}

\section{Family and Down syndrome: Stress, coping and family resources}

\begin{abstract}
This study aims to identify levels of stress and coping strategies of parents of children with Down syndrome (DS), as well as the adequacy of family resources. Fourteen families with a child with DS were visited. The parents filled out the questionnaire of family system characteristics, the inventory of stress, the coping strategies inventory and the scale of family resources. The results show that mothers $(n=7)$ and fathers $(n=6)$ present symptoms of stress in resistance and quasi exhaustion stages. The coping strategies used by parents are problem solving and social support. The data from family resources scale indicate that these are fairly adequate to the needs of these families. We highlight the importance of intervention programs for these families.
\end{abstract}

KEYWORDS: coping strategies, Down syndrome, family, social support, family resources

Educar uma criança com deficiência intelectual não é uma tarefa fácil e, invariavelmente, gera estresse na unidade familiar (Azar \& Badr, 2010; Kumar, 2008). A literatura demonstra que genitores de crianças com necessidades especiais apresentam maior nível de estresse parental que genitores de criança com desenvolvimento típico, sendo que uma variedade de estressores é associada à criação dos filhos (Dabrowska \& Pisula, 2010; Glidden, Billings, \& Jobe, 2006, Pereira-Silva, 2015; Rooke \& Pereira-Silva, 2016). O estudo de Keller e Honing (2004), por exemplo, demonstrou que o estresse materno está associado às demandas de cuidado com a criança e o estresse paterno, à dificuldade do pai em manter uma relação de proximidade com o filho. Além disso, o nível de estresse de pais e mães é influenciado pelas características da criança. Diante dessa condição, os genitores devem desenvolver mecanismos eficazes de enfrentamento para se adaptarem com sucesso à situação (Couto, 2017; Glidden et al., 2006; Gupta,
Sapra, \& Kabra, 2013; Rooke \& Pereira-Silva, 2016). Consideram-se bem-sucedidas aquelas famílias capazes de mobilizar seus recursos internos e externos de apoio para lidarem eficazmente com as necessidades do(a) filho(a) com deficiência, de forma que o bem-estar da família poderá ser preservado a depender das estratégias de enfrentamento dos membros familiares (Kumar, 2008; Oliveira, 2013; Rooke \& Pereira-Silva, 2016).

As estratégias de enfrentamento ou coping são habilidades desenvolvidas pela pessoa para o domínio e adaptação a situações estressoras (Savoia, Santana, \& Mejias, 1996). Os autores definem coping como uma resposta do indivíduo ao estresse, que pode ser comportamental ou cognitiva e cujo objetivo é aumentar, criar ou manter a sua percepção de controle pessoal. Folkman e Lazarus (1985) classificaram as estratégias de enfrentamento/coping como: confronto, afastamento, autocontrole, aceitação da responsabilidade, apoio social, fuga e esquiva, resolução de

*E-mail: naraliana.silva@ufjf.edu.br 
problemas e reavaliação positiva. Esses autores destacam duas funções dessas estratégias: modificar a relação entre a pessoa e o ambiente, controlando ou alternando o problema (enfrentamento centrado no problema), ou adequar a resposta emocional ao problema (enfrentamento centrado na emoção). No presente trabalho, os termos estratégias de enfrentamento e coping são utilizados como sinônimos para se referirem ao mesmo processo (de enfrentamento).

A literatura tem demonstrado que pais e mães de pessoas com deficiência intelectual utilizam uma variedade de estratégias de enfrentamento (Oliveira, 2013), não havendo consenso sobre o assunto até o momento. No estudo de Glidden et al. (2006), a estratégia mais utilizada por pais e mães biológicos e adotivos de crianças com atraso no desenvolvimento foi a resolução de problemas, e a menos utilizada foi a fuga-esquiva. Em consonância, os resultados de Barbosa e Oliveira (2008), com 10 mães e um pai de pessoas com deficiência intelectual, síndrome de Down (SD), microcefalia e autismo, demonstraram que a estratégia de enfrentamento mais utilizada foi a resolução de problemas e a menos utilizada foi o afastamento. Já no estudo de Oliveira (2013), genitores de adolescentes com SD utilizaram mais frequentemente fuga-esquiva, enquanto a estratégia menos utilizada pelas mães foi aceitação de responsabilidade e pelos pais, o confronto. Rooke e Pereira-Silva (2016), investigando famílias de crianças com SD, ressaltam que a estratégia mais utilizada pelas mães e pelos pais foi reavaliação positiva, enquanto a menos utilizada foi fuga-esquiva.

No estudo conduzido por Bingham, Correa e Huber (2012) as estratégias paliativas, tais como negação, questionamento e pensamento positivo, bem como as de resolução de problemas, foram as mais relatadas pelas mães de crianças com deficiência. Já os resultados de Moawad (2012) revelaram que as mães de crianças com deficiência enfrentam suas situações adversas e problemas utilizando estratégias como aquisição de apoio social e reformulação. No entanto, a busca por apoio espiritual foi a estratégia menos utilizada pelas participantes.

Apesar de ainda não haver consenso entre os estudos, Glidden et al. (2006) afirmam que os genitores que usam estratégias de enfrentamento focalizadas na resolução de problemas e apoio social têm apresentado resultados mais positivos, enquanto que aqueles que utilizam estratégias centradas na negação, esquiva e evitação têm demonstrado ajustes menos positivos. Por exemplo, Woodman e Hauser-Cram (2013) afirmam que, quando os genitores se concentram na resolução do problema gerador de estresse, há um efeito benéfico imediato sobre os sintomas de depressão, demonstrando que a estratégia de enfrentamento pode ter efeito sobre a saúde mental dos genitores.

Entre as estratégias de enfrentamento utilizadas pelas famílias, a busca por suporte social merece destaque pelo fato de as famílias com um filho(a) com deficiência terem uma sobrecarga adicional nos níveis social, psicológico e financeiro, em razão dos cuidados com a criança (Pereira-
Silva, Oliveira, \& Rooke, 2015; Shapiro, Blacher, \& Lopez, 1998). A literatura demonstra que o suporte social influencia direta e indiretamente vários aspectos do funcionamento familiar e parental, incluindo a sua adaptação ao estresse e ao bem-estar emocional (Felizardo, 2010).

No tocante ao estresse, os estudos têm mostrado que mães de crianças com deficiência intelectual experienciam mais estresse que os pais nesse mesmo grupo e também que as mães de crianças com desenvolvimento típico (Hastings, Daley, \& Burns, 2006; Shapiro et al., 1998). No entanto, há dados que mostram níveis similares de estresse em mães e pais de crianças com deficiência intelectual em idade escolar. $\mathrm{O}$ estresse em genitores de crianças com deficiência intelectual está associado aos seguintes fatores: temperamento da criança, isolamento, retirada social (Shapiro et al., 1998), status empregatício, satisfação conjugal (Gerstein, Crnic, Blacher, \& Baker, 2009), própria avaliação do genitor a respeito do funcionamento da família, satisfação com o apoio recebido dos profissionais (Dempsey, Keen, Pennell, O'Reilly, \& Neilands, 2009), apoio recebido de familiares e não familiares/informal (Gerstein et al., 2009) e bem-estar psicológico (Cramm \& Nieboer, 2011). Destaca-se que as estratégias de enfrentamento utilizadas pelos genitores podem ter um efeito direto em sua saúde mental e seu bem-estar.

Em relação aos recursos da família, a definição utilizada no presente trabalho é a indicada por Dunst e Leet (como citado por Dunst, Leet, \& Trivette, 1988), sendo compreendidos os recursos humanos e físicos, incluindo abrigo, alimento e transporte, os recursos financeiros, serviços de cuidado da criança (como babá e creche), tempo para estar com a família e com os amigos e cuidados com a saúde. Dunst et al. (1988) demonstraram que os recursos familiares influenciam o bem-estar e têm consequências positivas para a saúde de mães de crianças com atraso no desenvolvimento. Por outro lado, a inadequação de recursos familiares pode influenciar a baixa adesão das mães aos tratamentos prescritos ao filho com deficiência, provavelmente pelo fato de que mães nessa situação estão mais preocupadas em suprir necessidades básicas da família, como alimentação, moradia e saúde.

Nas últimas duas décadas, ocorreu um crescimento no número de pesquisas na área de família e deficiência com foco nas estratégias de enfrentamento utilizadas por mães de crianças com deficiência intelectual (Azar \& Badr, 2010). No entanto, ainda há uma escassez de estudos no Brasil, assim como há poucos trabalhos que focalizem o pai como participante. Também se observa uma escassez de estudos nacionais sobre o estresse dos genitores, a rede de apoio e os recursos familiares em famílias de pessoas com deficiência intelectual. Diante da importância social e científica de se investigarem famílias com crianças com $\mathrm{SD}$, o presente estudo tem os seguintes objetivos: (1) identificar níveis de estresse em genitores de crianças com SD; (2) descrever as estratégias de enfrentamento utilizadas; (3) identificar a adequação dos recursos familiares. 


\section{MÉTODO}

\section{Participantes}

Participaram deste estudo 14 famílias compostas por mãe, pai e filhos(as), sendo que um destes tinha o diagnóstico de SD. Todos os membros da família moravam juntos na área urbana $(\mathrm{n}=13)$ ou na área rural $(\mathrm{n}=1)$ de uma cidade do interior de Minas Gerais. As crianças com SD tinham idades que variavam de quatro a dez anos, com média de sete anos. Oito crianças eram do sexo masculino e seis, do sexo feminino. A média da idade dos pais foi de 45,8 anos e das mães, de 44,4 anos. As famílias tinham, em média, 2,14 filhos. A renda familiar foi de, em média, 5,57 salários mínimos à época da coleta de dados. As famílias serão identificadas pela letra $\mathrm{F}$ seguida por um número, por exemplo, F1, F2, F3.

\section{Instrumentos}

Para a coleta de dados foram utilizados o Questionário de Caracterização do Sistema Familiar (Dessen, 2009), o Inventário de Sintomas de Stress para Adultos (ISSL; Lipp, 2000), o Inventário de Estratégias de Coping (Savoia et al., 1996) e a Escala de Recursos da Família (Nunes, 2006). O Questionário de Caracterização do Sistema Familiar tem o objetivo de coletar informações sobre idade e escolaridade dos membros da família, profissão e estado civil dos genitores, renda familiar, a composição da rede de apoio social, entre outros aspectos. O ISSL mede a frequência de sintomas de estresse, o tipo de sintoma existente (se físico, psicológico ou ambos) e a fase de estresse em que o respondente se encontra. Apresenta um modelo quadrifásico, cuja primeira fase é a do alerta; a segunda, de resistência; a terceira, de quase exaustão; e a quarta, de exaustão. Quanto maior a pontuação no ISSL, maior a probabilidade de alcançar sintomas significativos indicativos de estresse e mais avançada é a fase deste. O Inventário de Estratégias de Coping é composto por 66 itens que descrevem pensamentos e ações que as pessoas utilizam para lidar com demandas internas ou externas de um determinado evento estressante. Os itens estão divididos de acordo com as estratégias de enfrentamento utilizadas: confronto, afastamento, autocontrole, suporte social, aceitação de responsabilidade, fuga-esquiva, resolução de problemas e reavaliação positiva. A versão adaptada por Savoia et al. (1996) é do original de Folkman e Lazarus (1985). A Escala de Recursos da Família é constituída por 29 itens que avaliam a adequação de recursos que têm sido identificados como principais componentes do suporte intra e extrafamiliar. Nessa escala, o genitor é solicitado a indicar o quão cada um dos recursos estão adequados para atender às necessidades da família. Os itens da escala são classificados em seis subescalas: crescimento e suporte, saúde e necessidades, necessidades físicas e abrigo, suporte intrafamiliar, cuidados com a criança e recursos pessoais. O instrumento proposto por Dunst e Leet (1987) foi traduzido por Nunes (2006), entretanto ainda não está padronizado para a população brasileira.

\section{Procedimentos}

O presente estudo foi aprovado por Comitê de Ética em Pesquisa. A Secretaria de Educação do município disponibilizou uma lista de escolas de educação infantil e ensino fundamental, tendo sido contatadas 26 delas. Destas, 13 não tinham crianças com SD matriculadas. Duas instituições de educação especial também foram contatadas. Essas instituições e escolas municipais foram visitadas para que pudessem possibilitar o contato com as famílias de crianças com SD para que fosse possível realizar o recrutamento e seleção das mesmas. Obteve-se o consentimento de 14 famílias para participação no estudo.

A coleta de dados foi realizada na residência das famílias em duas visitas. Na primeira visita, pai e mãe assinaram o Termo de Consentimento Livre e Esclarecido, a mãe respondeu ao Questionário de Caracterização do Sistema Familiar e ambos os genitores responderam, separadamente, ao ISSL. Na segunda visita, o Inventário de Estratégias de Coping foi respondido pela mãe e pelo pai, separadamente, e a mãe respondeu também à Escala de Recursos da Família.

Para análise de dados, utilizou-se estatística descritiva, especialmente, devido à natureza dos instrumentos e à quantidade de participantes. $\mathrm{Na}$ análise dos índices de estresse, seguiram-se os procedimentos constantes do Manual do ISSL (Lipp, 2000). Para análise dos dados da Escala de Recursos da Família, calculou-se o escore das subescalas, bem como o escore total, somando-se os pontos de acordo com uma escala do tipo Likert (nunca adequado $=1$, raramente adequado $=2$, algumas vezes adequado $=3$, geralmente adequado $=4$, sempre adequado=5). De acordo com as normas de correção da Escala de Recursos da Família, quanto maior é a pontuação, mais adequados são os recursos disponíveis à família, sendo o escore máximo de 145 pontos e total mínimo de 29. Para os itens que não se aplicam à família, indica-se a resposta não se aplica (NA). Por se tratar de um instrumento ainda não validado para a população brasileira, as análises ocorreram em função da comparação dos escores dentro do próprio grupo de participantes. Efetuou-se o cálculo da média de cada estratégia para cada genitor, seguindo os itens do Inventário de Estratégias de Coping. De acordo com esse instrumento, quanto maior a média, maior a frequência de uso daquela estratégia. Os itens estão divididos de acordo com as estratégias de enfrentamento utilizadas. 


\section{RESULTADOS}

Para verificar a adequação dos recursos da família na percepção da mãe, foram utilizadas a Escala de Recursos da Família e itens do Questionário de Caracterização do Sistema Familiar. A Tabela 1 apresenta a renda da família, a classificação econômica de acordo com a Associação Brasileira de Estudos Populacionais (ABEP, 2010) e os escores obtidos na Escala de Recursos da Família.

A média $(\overline{\mathrm{X}})$ dos escores totais é 117,14 e o desvio padrão $(\sigma), 12,95$. As médias obtidas em cada subescala foram: Crescimento e Suporte $\overline{\mathrm{X}}=26,14(\mathrm{~m}=25,5, \sigma=$ 6,17); Saúde e Necessidades $\bar{X}=32,64(m=35,0, \sigma=3,69)$; Necessidades Físicas e Abrigo $\bar{X}=36,0(m=36,0, \sigma=3,53)$; Suporte Intrafamiliar $\overline{\mathrm{X}}=8,43(m=8,5, \sigma=1,69)$; Cuidados com a Criança $\overline{\mathrm{X}}=5,29(m=5,0, \sigma=2,33)$; Recursos Pessoais $\bar{X}=8,64(m=9,0, \sigma=1,51)$. Não foram verificadas associações entre a renda familiar e o escore total da Escala de Recursos da Família. Na análise por família, verifica-se que o escore mais elevado foi obtido pela família F4 (136) e o mais baixo, pela família F1 (93). Destaca-se que seis famílias estão classificadas nas classes $\mathrm{C} 1$ e $\mathrm{C} 2$ e duas, na classe E, entretanto, F1 (classe C1) foi a que obteve escore mais baixo enquanto F6 (classe E) obteve escore elevado (130), mesmo de acordo com a renda familiar. Esse resultado permite afirmar que F4 considera os recursos disponíveis mais adequados para atender às suas necessidades, enquanto F1 considera os recursos menos adequados.

Destaca-se que 11 famílias na subescala Saúde e Necessidades obtiveram o escore máximo (35) ou próximo do máximo (32 e 34), indicando que essas famílias avaliam como adequados esses recursos. Para F1, F9 e F14, esses recursos mostraram-se menos adequados, uma vez que os escores variaram entre 25 e 29 pontos. Na subescala Necessidades Físicas e Abrigo, três famílias obtiveram escores entre 31 e 33, os quais foram os mais baixos, evidenciando que essas famílias não possuem esses recursos disponíveis de forma adequada. Por outro lado, a maioria das famílias avalia que suas necessidades físicas e abrigo são atendidos de forma satisfatória. Ressalta-se que os recursos disponíveis para satisfazer as necessidades de Cuidados com a Criança são avaliados com escore de 5 pontos pela maioria das mães ( $\mathrm{n}=8)$, escore máximo ( 10 pontos) por duas mães (F2, F4) e com escore mínimo (1 ponto) pela mãe da F1. Esse resultado indica que, para a maioria dessas famílias, ainda são escassos os recursos que os apoiam nos cuidados com seus filhos com SD. Os Recursos Pessoais foram avaliados pela maioria das mães $(\mathrm{n}=11)$ como disponíveis e adequados (entre 8 e 10 pontos).

No tocante à existência de sintomas de estresse em pais e mães, identificaram-se 15 genitores (mães: $\mathrm{n}=7$; pais: $\mathrm{n}=8$ ) que não apresentaram esses sintomas. Em relação àqueles com índices de estresse, 11 estavam na fase de resistência (mães: $n=6$; pais: $n=5$ ) e dois estavam na fase de quase exaustão (mães: $n=1$; pais: $n=1$ ). No que tange à sintomatologia, houve predominância de genitores com sintomas psicológicos (mães: $n=4$; pais: $n=3$ ). A Tabela 2 apresenta esses resultados.

No que tange à utilização das estratégias de enfrentamento, verificam-se que a mais utilizada pelos pais é a resolução de problemas e pelas mães, o suporte social. Reavaliação positiva, aceitação de responsabilidade, autocontrole e confronto também são estratégias utilizadas pelos genitores do presente estudo, com médias similares. Já a estratégia menos utilizada tanto por mães quanto por pais é fugaesquiva. $\mathrm{O}$ teste Mann-Whitney não demonstra diferença estatisticamente significativa entre as estratégias utilizadas por pais e mães. Os dados podem ser visualizados na Tabela 3.

Tabela 1

Dados Socioeconômicos e Escores das Subescalas de Recursos da Família

\begin{tabular}{|c|c|c|c|c|c|c|c|c|c|}
\hline \multirow[b]{2}{*}{ Família } & \multirow[b]{2}{*}{ Renda $^{\mathrm{a}}$} & \multirow[b]{2}{*}{$\begin{array}{c}\text { Classificação } \\
\text { ABEP }\end{array}$} & \multicolumn{7}{|c|}{ Subescalas de Recursos da Família } \\
\hline & & & $\begin{array}{c}\text { Crescimento e } \\
\text { Suporte } \\
\mathrm{EM}^{\mathrm{b}}=40\end{array}$ & $\begin{array}{c}\text { Saúde e } \\
\text { Necessidades } \\
\text { EM }=35\end{array}$ & $\begin{array}{c}\text { Necessidades } \\
\text { físicas e abrigo } \\
\text { EM }=40\end{array}$ & $\begin{array}{c}\text { Suporte } \\
\text { intrafamiliar } \\
\mathrm{EM}=10\end{array}$ & $\begin{array}{l}\text { Cuidados com a } \\
\text { criança } \\
\mathrm{EM}=10\end{array}$ & $\begin{array}{l}\text { Recursos } \\
\text { Pessoais } \\
\text { EM }=10\end{array}$ & $\begin{array}{c}\text { Escore total } \\
\mathrm{EM}=145\end{array}$ \\
\hline F1 & 4,70 & $\mathrm{C} 1$ & 22 & 25 & 31 & 7 & 1 & 7 & 93 \\
\hline F2 & 5,78 & B2 & 32 & 35 & 40 & 8 & 10 & 9 & 134 \\
\hline F3 & 1,06 & $\mathrm{E}$ & 18 & 35 & 40 & 9 & 5 & 10 & 117 \\
\hline $\mathrm{F} 4$ & 15,68 & B1 & 33 & 35 & 40 & 10 & 10 & 8 & 136 \\
\hline F5 & 2,83 & $\mathrm{C} 2$ & 30 & 35 & 35 & 10 & 5 & 9 & 124 \\
\hline F6 & 1,23 & $\mathrm{E}$ & 31 & 35 & 39 & 10 & 5 & 10 & 130 \\
\hline F7 & 9,60 & B1 & 27 & 35 & 40 & 5 & 6 & 10 & 123 \\
\hline F8 & 13,72 & B1 & 29 & 34 & 37 & 6 & 5 & 9 & 120 \\
\hline F9 & 3,22 & $\mathrm{C} 1$ & 22 & 25 & 32 & 10 & 5 & 7 & 101 \\
\hline F10 & 4,90 & $\mathrm{C} 1$ & 18 & 32 & 36 & 10 & 3 & 8 & 107 \\
\hline F11 & 4,02 & $\mathrm{C} 1$ & 22 & 32 & 36 & 7 & 5 & 8 & 110 \\
\hline F12 & 9,80 & B1 & 38 & 35 & 30 & 10 & 5 & 10 & 128 \\
\hline F13 & 19,80 & A2 & 24 & 35 & 33 & 8 & 4 & 7 & 111 \\
\hline F14 & 2,12 & $\mathrm{C} 2$ & 20 & 29 & 35 & 8 & 5 & 9 & 106 \\
\hline
\end{tabular}

Nota. ABEP = Associação Brasileira de Estudos Populacionais.

${ }^{\mathrm{a}}$ Renda familiar em salários mínimos à época da coleta de dados. ${ }^{\mathrm{b}} \mathrm{EM}=$ Escore Máximo. 
Ao realizar a comparação entre as estratégias de enfrentamento utilizadas por genitores com e sem estresse, o teste Mann-Whitney identificou diferença significativa somente para as estratégias reavaliação positiva $(p=0,0279)$ e fuga-esquiva ( $\mathrm{p}=0,0331$ ), a um nível de significância de $5 \%$, indicando que genitores com estresse utilizam essas estratégias mais que genitores sem estresse, conforme pode ser visualizado na Tabela 4.

Tabela 2

Sintomatologia e Fases do Estresse Segundo Pais e Mães

\begin{tabular}{ccccc}
\hline \multirow{2}{*}{$\begin{array}{c}\text { Sintomatologia } \\
\text { do estresse }\end{array}$} & \multicolumn{3}{c}{ Fases do estresse } & \multicolumn{2}{c}{ Quase-exaustão } \\
\cline { 2 - 5 } & Pais & Resistência & Pais & Mães \\
\hline Físico & 1 & 2 & 1 & - \\
Psicológico & 3 & 3 & - & 1 \\
Físico e Psicológico & 1 & 1 & - & - \\
Total & 5 & 6 & 1 & 1 \\
\hline
\end{tabular}

Tabela 3

Estratégias de Enfrentamento Utilizadas pelos Genitores

\begin{tabular}{|c|c|c|c|c|c|c|c|}
\hline \multirow{3}{*}{$\begin{array}{l}\text { Estratégias de } \\
\text { enfrentamento }\end{array}$} & \multicolumn{4}{|c|}{ Genitores } & \multirow{2}{*}{\multicolumn{2}{|c|}{ Total }} & \\
\hline & \multicolumn{2}{|c|}{ Pais } & \multicolumn{2}{|c|}{ Mães } & & & \\
\hline & $\bar{X}$ & SD & $\bar{X}$ & SD & $\bar{X}$ & $\mathrm{SD}$ & $\begin{array}{c}\text { Teste U de } \\
\text { Mann-Whitney }\end{array}$ \\
\hline Resolução de Problemas & 2,02 & 0,64 & 1,99 & 0,82 & 2 & 0,72 & 0,889 \\
\hline Suporte Social & 1,9 & 0,62 & 2,03 & 0,66 & 1,98 & 0,63 & 0,694 \\
\hline Reavaliação Positiva & 1,68 & 0,39 & 1,79 & 0,36 & 1,74 & 0,38 & 0,414 \\
\hline $\begin{array}{l}\text { Aceitação de } \\
\text { responsabilidade }\end{array}$ & 1,5 & 0,61 & 1,86 & 0,67 & 1,68 & 0,66 & 0,178 \\
\hline Autocontrole & 1,61 & 0,36 & 1,62 & 0,64 & 1,62 & 0,50 & 0,982 \\
\hline Confronto & 1,54 & 0,42 & 1,51 & 0,57 & 1,53 & 0,49 & 0,926 \\
\hline Afastamento & 1,17 & 0,40 & 1,04 & 0,50 & 1,1 & 0,46 & 0,237 \\
\hline Fuga-esquiva & 0,7 & 0,39 & 0,8 & 0,54 & 0,75 & 0,47 & 0,729 \\
\hline
\end{tabular}

$N=14$ pais; 14 mães.

Tabela 4

Estratégias de Enfrentamento Utilizadas pelos Genitores com e sem Estresse

\begin{tabular}{|c|c|c|c|c|c|c|c|}
\hline \multirow{3}{*}{ Estratégias } & \multicolumn{4}{|c|}{ Genitores } & \multirow{2}{*}{\multicolumn{2}{|c|}{ Total }} & \multirow[b]{3}{*}{$\begin{array}{c}\text { Teste U de } \\
\text { Mann-Whitney }\end{array}$} \\
\hline & \multicolumn{2}{|c|}{ Sem estresse } & \multicolumn{2}{|c|}{ Com estresse } & & & \\
\hline & $\bar{X}$ & $\mathrm{SD}$ & $\bar{X}$ & $\mathrm{SD}$ & $\bar{X}$ & SD & \\
\hline Resolução de Problemas & 1,89 & 0,84 & 2,13 & 0,58 & 2,00 & 0,73 & 0,516 \\
\hline Suporte Social & 1,82 & 0,69 & 2,15 & 0,54 & 1,98 & 0,63 & 0,171 \\
\hline Reavaliação Positiva & 1,58 & 0,38 & 1,91 & 0,30 & 1,73 & 0,38 & $0,028 *$ \\
\hline Aceitação de responsabilidade & 1,60 & 0,61 & 1,77 & 0,72 & 1,68 & 0,66 & 0,576 \\
\hline Autocontrole & 1,55 & 0,64 & 169 & 0,30 & 1,62 & 0,51 & 0,561 \\
\hline Confronto & 1,42 & 0,55 & 1,64 & 0,42 & 1,52 & 0,49 & 0,295 \\
\hline Afastamento & 1,00 & 0,46 & 1,22 & 0,44 & 1,10 & 0,46 & 0,329 \\
\hline Fuga-esquiva & 0,57 & 0,39 & 0,96 & 0,47 & 0,75 & 0,47 & $0,033 *$ \\
\hline
\end{tabular}

$* \mathrm{p}<0,05 . \mathrm{N}=15$ genitores sem estresse; 13 genitores com estresse.

\section{DISCUSSÃO}

As estratégias de enfrentamento e o estresse são fatores importantes que interferem nas relações familiares, bem como na dinâmica e no funcionamento de famílias com filhos com deficiência intelectual ou com SD. Os resultados indicam que pais e mães utilizam estratégias similares e adequadas, conforme também constataram Glidden et al. (2006) e Barbosa e Oliveira (2008). Entretanto, genitores com estresse tendem a utilizar mais as estratégias de reavaliação positiva e fuga-esquiva do que os genitores sem estresse, indicando não haver equilíbrio na utilização de estratégias para o enfrentamento de situações estressantes, dificuldades ou adversidades pelos genitores com estresse. Provavelmente a oscilação na utilização dessas estratégias seja um indicativo de dificuldades emocionais, evidenciadas na prevalência de sintomas psicológicos de estresse. Essas evidências corroboram os dados encontrados em outros estudos acerca das implicações do estresse para a saúde mental dos genitores com filhos com deficiência intelectual ou com SD (Bingham et al., 2012; Cramm \& Nieboer, 2011). 
As estratégias de resolução de problemas e suporte social prevaleceram entre as mais utilizadas pelos genitores deste estudo. Outras investigações também encontraram resultados similares (Glidden et al., 2006; Moawad, 2012). Apesar de a maioria dos genitores adotar estratégias de enfrentamento adequadas, vale ressaltar que aqueles com estresse também fazem uso da estratégia fuga-esquiva. Woodman e HauserCram (2013) afirmam que determinadas estratégias exercem maior ou menor impacto sobre o bem-estar dos genitores, principalmente, de mães de adolescentes com problemas de comportamento, sugerindo a implementação de intervenções com foco no treinamento de estratégias adequadas. Destacase, portanto, a necessidade de planejamento de programas de educação familiar, baseados em pressupostos sistêmicos, que possam dar suporte e orientação às famílias com filhos com SD (Pereira-Silva \& Dessen, 2005).

A literatura tem apontado que o estresse dos genitores está associado a diferentes fatores e não somente ao fato de se ter um filho com SD ou com deficiência intelectual (Cramm \& Nieboer, 2011; Dempsey et al., 2009; Gerstein et al., 2009; Shapiro et al., 1998). Os resultados encontrados no presente estudo mostram não haver diferenças importantes, no grupo de genitores estressados, entre pais e mães quanto à fase do estresse em que se encontram (resistência) e ao tipo de sintoma (psicológico). Esse resultado não corrobora a literatura na área, a qual aponta que o estresse está mais presente em mães do que em pais (Hastings et al., 2006; Pereira-Silva \& Dessen, 2006; Shapiro et al., 1998). Destacase a importância de implementação de mais estudos nessa área, com número maior de genitores, associando o estresse a outros fatores que são destacados como influentes nos sintomas de estresse, tais como o apoio social (Gerstein et al., 2009) e a qualidade da relação conjugal. Estudos brasileiros focalizando a associação entre esses fatores são escassos.

Os resultados sobre a adequação dos recursos indicam que, no presente estudo, as mães avaliam seus recursos como sendo mais adequados do que as mães no estudo de Oliveira (2013). Destaca-se que a maioria das mães avalia como apropriado o acesso à moradia, à alimentação, ao emprego, à saúde e aos serviços públicos em geral, conforme apontam os escores nas subescalas. Esses recursos parecem ser os essenciais para proporcionar e manter satisfatórias as condições de vida das famílias. Por outro lado, aqueles recursos que propiciam os cuidados com o filho com SD não estão disponíveis durante todo o mês. Destaca-se que a família F1 é aquela que possui os recursos em menor quantidade, isto é, estes não estão disponíveis durante todo o mês, podendo ter impactos importantes na dinâmica da família, bem como no bem-estar dos genitores, na qualidade da relação conjugal e, também, no desenvolvimento do filho com SD. Nessa família, a mãe apresenta sintomas de estresse e o pai não, sendo que ambos utilizam estratégias de enfrentamento adequadas.

As famílias com renda entre um e dois salários mínimos (F3, F6) apresentaram escore total adequado na Escala de Recursos da Família, indicando que as mães avaliam que os recursos estão adequados para atender as necessidades de suas famílias. Não foram verificadas associações entre a renda familiar e o escore total da Escala de Recursos da Família. Os resultados do presente estudo divergem daqueles encontrados por Nunes (2006), em que as famílias com menores escores nessa escala eram também as famílias com menor nível socioeconômico. Considera-se importante a investigação da variável recursos da família, uma vez que esta pode interferir na possibilidade de as famílias oferecerem atendimentos a suas crianças com SD.

Os dados do presente estudo indicam que essas famílias, apesar de apresentarem sintomas de estresse, tanto pais como mães, têm utilizado, no geral, estratégias de enfrentamento adequadas quando lidam com situações-problema. $\mathrm{O}$ estresse avaliado nesta investigação revela o estresse em relação à vida e seus eventos e o mais interessante é avaliar, sobretudo, o estresse parental, aquele que é relativo às funções parentais. Assim, não se deve atribuir o estresse necessariamente à parentalidade. Por outro lado, os dados evidenciam a necessidade da implementação de programas de intervenção com foco nas famílias. Um programa de intervenção, portanto, deve envolver a família toda, principalmente porque seus benefícios são extensivos ao modo de organização e funcionamento familiar, especialmente no que se refere aos recursos psicológicos e à qualidade das interações entre seus membros (Pereira-Silva \& Dessen, 2005).

\section{REFERÊNCIAS}

Azar, M., \& Badr, L. K. (2010). Predictors of coping in parents of children with an intellectual disability: Comparison between lebanese mothers and fathers. Journal of Pediatric Nursing, $25,46-56$.

Barbosa, A. J. G., \& Oliveira, L. D. (2008). Estresse e enfrentamento em pais de pessoas com necessidades especiais. Psicologia em Pesquisa, 2, 36-50.

Bingham, A., Correa, V. I., \& Huber, J. J. (2012). Mothers' voices: Coping with their children's initial disability diagnosis. Infant Mental Health Journal, 33, 372-385.

Couto, D. L. (2017). A perspectiva de cuidadores primários acerca do diagnóstico da síndrome de Down e o processo de adaptação da família nos primeiros anos de vida da criança (Dissertação de Mestrado). Universidade Federal de Minas Gerais, Belo Horizonte, MG, Brasil.

Cramm, J. M., \& Nieboer, A. P. (2011). Psychological well-being of caregivers of children with intellectual disabilities: Using parental stress as a mediating factor. Journal of Intellectual Disability Research, 15(2), 101-113.

Dabrowska, A., \& Pisula, E. (2010). Parenting stress and coping styles in mothers and fathers of pre-school children with autism and Down syndrome. Journal of Intellectual Disability Research, 54, 266-280. 
Dessen, M. A. (2009). Questionário de caracterização do sistema familiar. In L. Weber \& M. A. Dessen (Orgs.), Pesquisando a família: Instrumentos para coleta e análise de dados (pp.102114). Curitiba: Juruá.

Dempsey, I., Keen, D., Pennell, D., O’Reilly, J., \& Neilands, J. (2009). Parent stress, parenting competence and familycentered support to young children with an intellectual or developmental disability. Research in Developmental Disabilities, 30, 558-566.

Dunst, C. J., \& Leet, H. E. (1987). Measuring the adequacy of resources in households with young children. Child: Care, Health, and Development, 13, 111-125.

Dunst, C. J., Leet, H., \& Trivette, C. M. (1988). Family resources, personal well-being and early intervention. Journal of Special Education, 22, 108-116.

Felizardo, S. M. A. S. (2010, fevereiro). Os efeitos do suporte social em famílias de crianças com deficiência. Trabalho apresentado no VII Simpósio Nacional de Investigação em Psicologia, Universidade do Minho- Portugal.

Folkman, S., \& Lazurus, R. S. (1985). If it changes it must be a process: Study of emotion and coping during three stages of a college examination. Journal of Personality and Social Psychology, 48, 150-170.

Gerstein, E. D., Crnic, K. A., Blacher, J., \& Baker, B. L. (2009). Resilience and the course of daily parenting stress in families of young children with intellectual disabilities. Journal of Intellectual Disability Research, 53, 981-997.

Glidden, L. M., Billings, F. J., \& Jobe, B. M. (2006). Personality, coping style and well-being of parents rearing children with developmental disabilities. Journal of Intellectual Disability Research, 50, 949-962.

Gupta, N., Sapra, S., \& Kabra, M. (2013). Coping strategies of parents of Down syndrome children in India. The Indian Journal of Pediatrics, 80, 534-535.

Hastings, R. P., Daley, D. \& Burns, C. (2006). Maternal distress and expressed emotion: Cross-sectional and longitudinal relationships with behavior problems of children with intellectual disabilities. American Journal on Mental Retardation, 11, 48-61.

Keller, D., \& Honig, A. S. (2004). Maternal and paternal stress in families with school-aged children with disabilities. American Journal of Orthopsychiatry, 24, 337-348.

Kumar, G. V. (2008). Psychological stress and coping strategies of the parents of mentally challenged children. Journal of the Indian Academy of Applied Psychology, 34, 227-231.

Lipp, M. N. (2000). Inventário de Sintomas de Stress para Adultos de Lipp. São Paulo: Casa do Psicólogo.

Moawad, G. E. N. A. (2012). Coping strategies of mothers having children with special needs. Journal of Biology, Agriculture and Healthcare, 2, 77-84.

Nunes, C.C. (2006). Interação entre irmãos de indivíduos com deficiência mental: O papel da idade e do apoio social da família (Dissertação de Mestrado). Universidade Federal de São Carlos, São Carlos, SP, Brasil.

Oliveira, L. D. (2013). Famílias de adolescentes com deficiência intelectual: Estresse, estratégias de enfrentamento e apoio social (Dissertação de Mestrado não publicada). Universidade Federal de Juiz de Fora, Juiz de Fora, MG, Brasil.

Pereira-Silva, N. L. (2015). Estresse e ajustamento conjugal em famílias com filho(a) com síndrome de Down. Interação Psicologia, 19(2), 225-234.

Pereira-Silva, N. L., \& Dessen, M. A. (2005). Intervenção precoce e família: Contribuições do modelo bioecológico de Bronfenbrenner. In M. A. Dessen \& A. L. Costa Junior (Orgs.), A ciência do desenvolvimento humano: Tendencias atuais e perspectivas futuras (pp. 152-167). Porto Alegre: Artmed.

Pereira-Silva, N. L., \& Dessen, M. A (2006). Padrões de interação genitores-crianças com e sem síndrome de Down. Psicologia: Reflexão e Crítica, 19, 283-291.

Pereira-Silva, N. L., Oliveira, L., \& Rooke, M. I. (2015). Famílias com adolescente com síndrome de Down: Apoio social e recursos familiares. Avances en Psicología Latinoamericana, 32, 269-283.

Rooke, M. I., \& Pereira-Silva, N. L. (2016). Indicativos de resiliência familiar em famílias de crianças com síndrome de Down. Estudos de Psicologia, 33(1), 117-126.

Savoia, M. G., Santana, P., \& Mejias, N. P. (1996). Adaptação do Inventário de Estratégias de Coping de Folkman e Lazarus para o Português. Revista de Psicologia USP, 6, 183-201.

Shapiro, J., Blacher, J., \& Lopez, S. R. (1998). Maternal reactions to children with mental retardation. In J. A. Burack, R. M. Hodapp, \& E. Zigler (Orgs.), Handbook of mental retardation and development (pp. 606-636). Cambridge: Cambridge University Press.

Woodman, A. C., \& Hauser-Cram, P. (2013). The role of coping strategies in predicting change in parenting efficacy and depressive symptoms among mothers of adolescents with developmental disabilities. Journal of Intellectual Disability Research, 57, 513-530. 